\title{
Smart Adoption System using Image Processing Techniques
}

\author{
G.Shobana, M.Suguna, C.Umamageshwari
}

\begin{abstract}
Adoption of child plays a major role in recent times. This is due to the emotional and ethical challenges faced by both the adopting parent and the children.This paper presents a new technique of applying image processing technology that uses an image based approach to the system. With the rapid evolution of technology there are large numbers of face recognition algorithm. In this paper we have attempted to review the best face recognition algorithm by considering all the algorithm and its functions. Viola Jones algorithm is used to develop the entire system. Using this algorithm image processing is implemented for further enhancement of the system .This lowers the complexity in the child adoption system and also helps to increase the efficiency of the system.
\end{abstract}

Keywords-algorithm, pixels, face, images, tools, accuracy, facial features, pre-processing, database matching

\section{INTRODUCTION}

Image processing is one of the most interesting and challenging technology in real time applications. It enhances the system perception level by making it a human perception system. With the rapid development of technology, artificial intelligence, pattern and face recognition it has many real time application in security, social media and

Access control. This technology can also be embedded with child adoption system in order to increase the system efficiency. By using facial recognition one can find and adopt the child with similar features. Image processing and MATLAB tool are very useful in this facial recognition. Using Viola Jones algorithm we can compare the image of father and mother with the child. The child whose match is approximate to the parent is allocated to them. Matching is a sensitive and complex task in the case of child adoption. Our proposed system provides the extraction of skin tone, other facial feature and provides accuracy as a result for the images of both parents and the child that is to be adopted. The difference of the calculated accuracy is found from the parents and the child. The child whose accuracy difference is less with that of parents is recognized and allocated for adoption. This is the back-end process that takes place before giving the matched result. This process needs an input image, MATLAB tool and viola Jones algorithm for performing the image processing technique which brings an innovative application for child adoption system in a smart way.

To summarize the main steps in this algorithm it consists of six basic steps 1) Input image (image of parents both father and mother) 2) Pre-processing (removal of noise) 3)

\footnotetext{
Revised Manuscript Received on August 14, 2019.

G.Shobana, Assistant Professor, Dept of CSE, Kumaraguru College of Technology, Coimbatore, T.N, India. (Shobana.g.cse@kct.ac.in)

Dr.M.Suguna, Assistant Professor, Dept of CSE, Kumaraguru College of Technology, Coimbatore, T.N, India. (Suguna.m.cse@kct.ac.in)

C.Umamageshwari, UG Scholar, Dept of CSE, Kumaraguru College of Technology, Coimbatore, T.N, India.
}

Feature-extraction (face, eyes, nose, mouth, skin-tone) 4) Accuracy-calculation (Euclidean-distance) 5) Matching (child image from database) 6) Result (child allocated for adoption)

This paper contains four sections, the first one describes the problem statement, the second section describes the approaches for image processing technology, the third section describes the future work and the fourth section concludes this paper.

\section{EXISTING SYSTEM}

Swati Chauhan, Dr. S. M. Ghosh (2017) had analysed on the images of face taken with different expressions of face with the help ofthe digital camera. The steps involved in Face detection: (1) Face image from 13 megapixel camera (2) Image pre-processing in Photoshop (512*512 pixels) (3) Image resize in MATLAB ( $8 * 8$ pixels) (4) $\mathrm{X}, \mathrm{Y}$ node values in table (5) Create histogram graph. The algorithms used in this paper are the method of geometrics and Skin Colour Modelling. This techniquegives the upper part face accuracy and enhances the time complexity. Both the model gives the images with filter in term of pixels value to get the position of afce that are more efficient for large image database.A graph of every face imagesare created by the joint histogram graph .It is described by a particular selection of a value. Finally comparison is based on graph[1].

M Iqtait, F S Mohamad, M Mamat (2018) had proposed the algorithm Active Shape Model (ASM) for matching with a collection of model points to an image which was restricted by a statistical shape model, and Active Appearance Model (AAM) to match both the model points position and texture representation of the object to an image. AAM algorithm consists of 3procedures: (i) connection of shape and texture vectors to every AAM in the training set, with that shape and texture vectors are connected in the same vector (ii) Computing the correlation coefficient matrix about thejoined shape and texture vectors in training set. (iii) Correlation coefficient matrix is analysed by utilizing PCA. [2]

DeepikaGarg, Anubhav Kumar Sharma (2015) have practised different extraction methods for the recognition of face: (a) generic methods (b) template-basedon feature methods, (c) matching of structure methods. Kanade is one of the algorithms for feature extraction in automatic in a quite simple manner. Kanade's approach involves the conversion of regular image into a binary image which later was proposed as an input to the program of extraction. The extraction program helps to find the various features in the 
face. The steps involved in feature extraction are Face detection, face location in the image, extraction of Feature/component,features like eyes, nose or chins, Recognition, comparing images. The outcome of this process will be the image which is analogous to the input images. [3]

PriyaGuptaa et all (2018) have practiced the FR method which was tested on Yale face database with black and white images of 15 samples, each having 11 images withvarious expressions making a total of 165 images. Images for each subject in different facial expression or configuration are: with glasses, happy,sad, without glasses, normal, sleepy, surprised, and wink etc. The categories are also called as subjects along with labels. The dataset is divided into 2 parts: (a) 148 images for training, (b) seventeen for testing. Softmax layer is used for final average accuracy by checking the number of correctly identified test samples .The idea implementation is on Python and version 3.5.3 (64 bit) system. For pre-processing we use Opencv package . [4]

Ashish R. Dandekar,S. M. Sakharkar, M.S. Nimbarte (2016), Identification is based on five prime stages. Input images: 3 frontal Images of the parents(Father and Mother) and images of the child are taken from dataset. Preprocessing: Variation in ill-luminance are corrected using Tan and Trigg method and cropping of image is done based on requirements. Facial feature extraction: Key image is used to select the interesting region. The purpose is to select the most favorable features that can achieve the best accuracy result.Classification of Feature: Similar features are identified in this stage. Adaboost algorithm is commonly practised feature selection and the learning algorithm which combines different weak classifiers to form a strong classifier by adjusting the weights of training samples at eachrepitition. It generates match scores based on the feature extraction from every facial segment.Verification: Kin relationship algorithm is used for verification. It is done based on the facial features comparision or facial featurematching. K-nearest-neighbor (KNN) algorithm with Euclidean metric distance was applied for verification to find the facial featurematched.[5]

JawadNagi, et al (2015) have used face recognition technique which use the approachbased on image towards artificial intelligence by eliminating repeated data through the compression ofimageusing the 2-dimensional discrete cosine transform (2D-DCT). In the $1^{\text {st }}$ stage, $2 \mathrm{D}$-DCT for every face image is computed, and feature vectorsare is formed from the discrete cosine transform (DCT)coefficients. The $2^{\text {nd }}$ stage practises a self-organizing $\operatorname{map}(\mathrm{SOM})$ with an unsupervised learning technique to differentiate the vectors into groups to recognize if the subject in the inputimage is "present" or "absent" in the database of image. [6]

Sujata.G et al (2016) have usedPrincipal Component Analysis (PCA) also called as Eigen face approach which describes the feature space and used to decrease theoriginal data space dimensionality. Linear Discriminant Analysis (LDA) is used for the selection of feature in methodsbased on the appearance and increases the discriminating capability of feature selection. Component analysis is not dependent for finding underlying characters or components from various dimensional statistical data.[7]

SmritiTikoo, Nitin Malik (2016) have proposed the system that uses the images of a person's face has been collected by taking 3 various samples of the person for the experiment. Every image is partitioned into 2 equal parts to exhibit the process of segmentation. Feed Forward Back Propagation neural network have been helped to train, test and validate the network for every part of the image using MATLAB. The histogram equivalent of binary and gray images had been calculated as the data to check for the process of recognitionusing performance plot, regression plot as means of parameters forthe performancecheck. [8]

Dr.Mridul Kumar Mathur, PriyankaBhati (2017) In this approach objects are detected through these 4 important methods:

a) Simple rectangular components-Haar similar factors.

b)Complete images for quick feature exposure.

c) AdaBoost ML method.

d) Cascade classifier to integrate various features accurately. [9]

Mohammad Ashraful Islam et al (2017) have proposed the system that benefits the KLT algorithm which involves the spatial common intensity transformation to oversee the deep search for the position that displays the match accuracy. It is much quicker than other common techniques for checking far hardly any potential matches between the pictures. Viola-Jones has a good detection rate in each scenario and is better than the Kanade Lucas-Tomasi in every sequence of events. [10]

\section{PROPOSED WORK \& RESULTS}

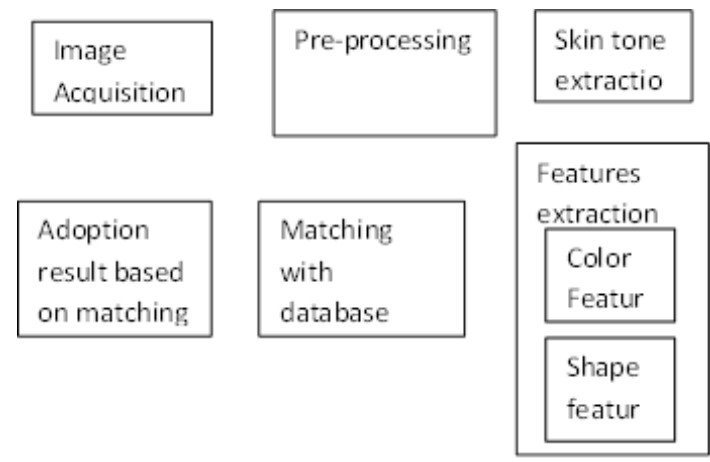

Fig 3.1.Architectural Diagram

Figure 3.1represents the architectural model of the recommended system in which images of the parents is taken as input and matches of child are made by imaging technique to adopt the child.

\section{A. Image Acquisition}

Image Acquisition is a process of getting an input image for the process of automatic adoption system based on image processing techniques which match the parents and child skin tone and features.

\section{B. Pre-processing}

The lowest level of abstraction is the preprocessing. The

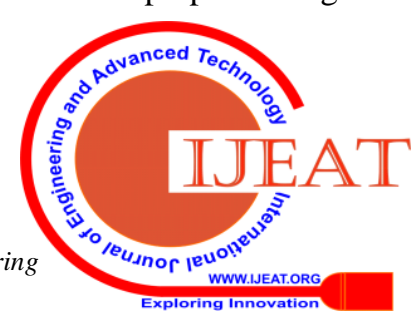


objective of preprocessing is to improve the dataimagewhich suppress the noise image distortions of data or increase some image features essential for further processing.

Data preprocessing illustrates any type of processing executed on raw data is used to prepare it for one more processing procedure. It is helped as a preliminary data mining practice, for data preprocessing which transforms the data into a format which will be more easily and accuaractely processed for theuserpurpose. There arevarious tools and methods available for preprocessing: (a)sampling, that selects a representative subset from the large set of data and transformation, which manipulates raw data to produce only 1 input and(b) de-noising, which removes noise from the data; (c)Normalization, which organizes the data for more efficient access and (d) feature extraction, which pulls out somespecific data.In Figure 3.2 original images are resized according to their pixel size with the databases.

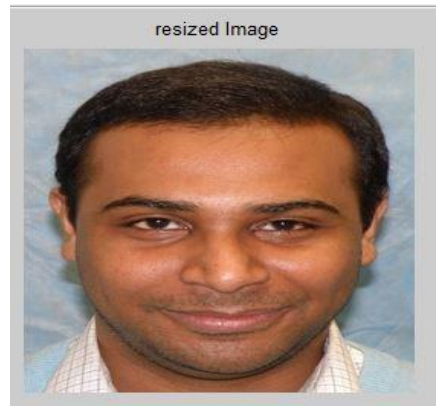

Fig 3.2.Re-sizing

\section{Feature Extraction}

The Viola-Jones algorithm is the initial object detection framework to provide competitive detection rates for the object. The cascade object detector utilises this algorithm for detectingpeople's faces, noses, eyes,mouth, or upper body. It is a method for object detection whichminimizes computation time whileachieving high detection rate effecticely. It is used for face detection in real time. The accuracy of Viola Jones algorithm enhances by removing or minimizing false detection of features.

Pattern recognition and featureextractioncommences from an basic set of data measured and builds the values which are derivedthat is featuresintended to be more informative,knowledgeable and non-redundant, facilitating the subsequent learning and generalization steps, and it leads to improved human interpretations. Feature extraction is analogous to D-reduction(dimensionality reduction).

When the data in the input is very large to be processed and it is suspected to be repititive then it can be transformed into a reduced set of features. Discovering a subset of the initial features is called feature selection.

Feature extraction describes the particular shape information present in a pattern so that the process of classifying the pattern is made simple by a usual procedure. Feature extraction is concluded after the preprocessing phase in recognition system for character. The fundamental task of pattern recognition is to take an input pattern and assign it as 1 of the possible output classes. This procedure can be divided into 2 general stages: Feature selection and Classification.

From the resized images, figure 3.3 face and eyes are extracted. Nose detection and mouth detection are shown in the figure 3.4. The facial features are extracted in the figure 3.5

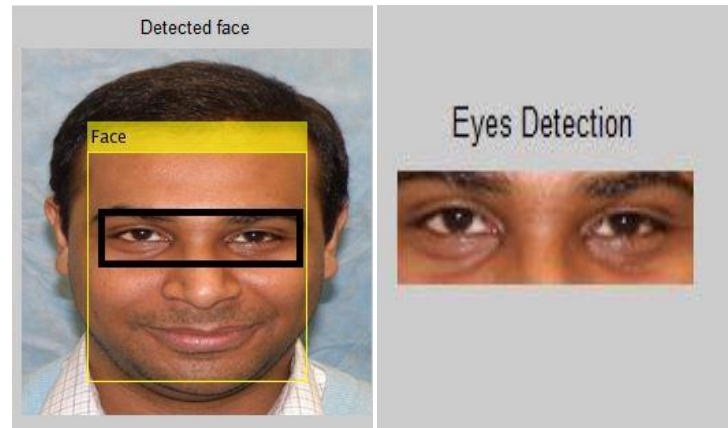

Fig 3.3.Face Detection and Eyes Detection

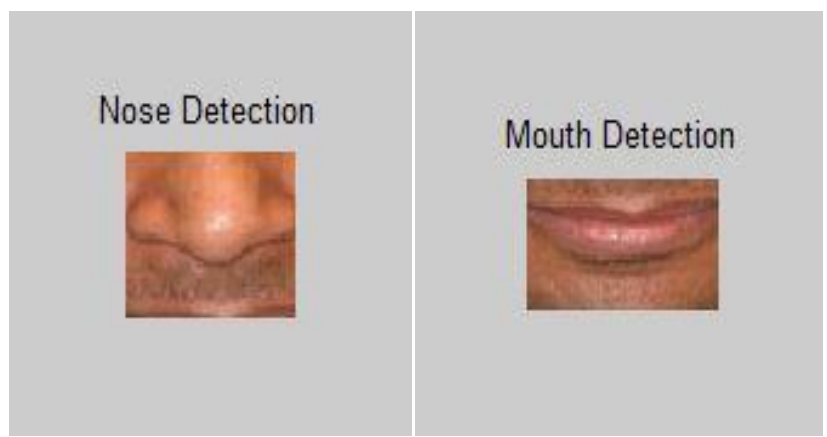

Fig 3.4.Nose Detection and Mouth Detection

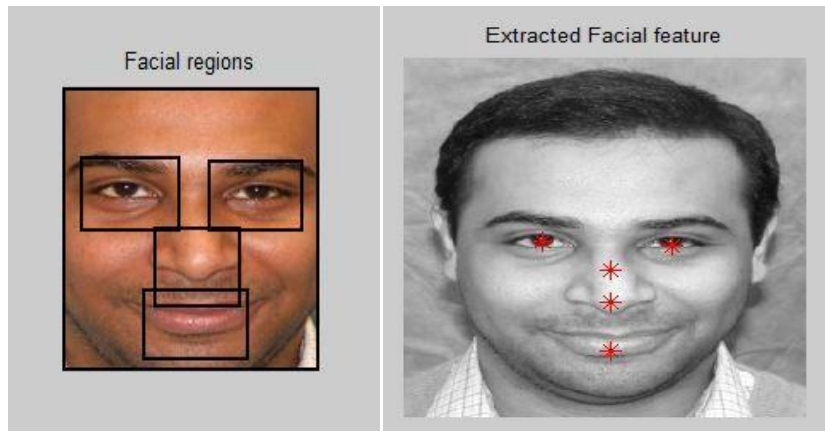

Fig 3.5.Facial Detection and .Extracted facial Features

\section{Skin tone extraction}

The main aim of the detection of skin color is to build a decision rule which will differentiate skin pixels and nonskin pixels. To analyse the skin colored pixels we need to discover the range of values that is most skin pixels would fall in a provided color space. The purpose of a color space is to facilitate the color specification in some standard. specification of a coordinate system and subspace within a system is a color space where every color is represented by a single point. Skin tone extraction is given in the figure 3.6 


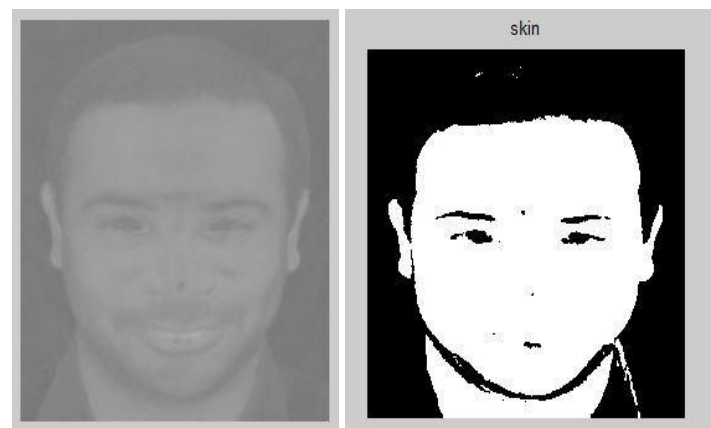

Fig3.6 Skin tone extraction

\section{E.Database Matching}

The extracted features are compared with the database features and finding the matches using Euclidean Distance based similarity measurement and give the adoption result for efficiently chose a parent for child as shown in figure 3.6

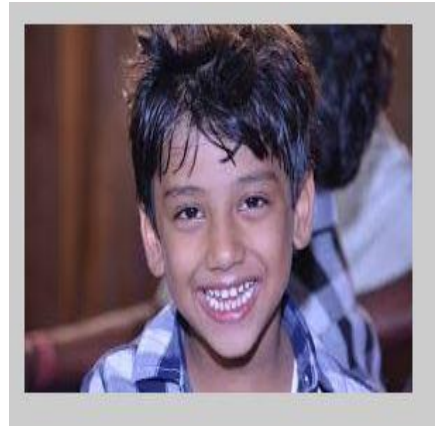

Fig 3.6 Matching result

\section{CONCLUSION AND FUTURE WORK}

AsViola Jones algorithm are used to extract features and skin tone of parents as well as the child. The images of mother, father and child is taken from database, then the minimum distance is found using Euclidean distance algorithm. Based on the minimum distance, the child will be allocated to the parents. This research also used for various field like biometric authentication, gesture reorganization .In the future work this algorithm can be combined with web application.

\section{REFERENCES}

1. Swati Chauhan, Dr. S. M. Ghosh, "A Method for Two Face Detection and Comparison using Image Processing with MATLAB Techniques", International Journal on Recent and Innovation Trends in Computing and Communication ISSN: 2321-8169, Volume: 5 Issue: 570 - 77.

2. M Iqtait, F S Mohamad, M Mamat, "Feature extraction for face recognition via Active Shape Model (ASM) and Active Appearance Model (AAM)",IORA-ICOR 2017 IOP Publishing IOP Conf. Series: Materials Science and Engineering 332 (2018) .

3. DeepikaGarg, Anubhav Kumar Sharma, "Face Recognition",IOSR Journal of Engineering (IOSRJEN) ISSN: 2250-3021 Volume 2, Issue 7(2015), PP 128133 www.iosrjen.org.

4. Dr.PriyaGuptaa, NidhiSaxenaa, MeetikaSharmaa, JagritiTripathia, "Deep Neural Network for Human Face Recognition",I.J. Engineering and Manufacturing, 2018, 1, 63-71 Published Online January 2018 in MECS .

5. Ashish R. Dandekar,S. M. Sakharkar, M.S. Nimbarte, "Identify Whether faces of Child is more closer to Father or Mother",International Journal of Recent Advances in
Engineering \& Technology (IJRAET),ISSN: 2347 - 2812, Volume-4, Issue -7, 2016

6. Jawad Nagi, Syed Khaleel Ahmed, Farrukh Nagi, "A MATLAB based Face Recognition System using Image Processing and Neural Networks",4th International Colloquium on Signal Processing and its Applications.

7. Sujata G. Bhele and V. H. Mankar, "A Review Paper on Face Recognition Techniques",International Journal of Advanced Research in Computer Engineering \& Technology (IJARCET) Volume 1, Issue 8, October 2016.

8. Smriti Tikoo, Nitin Malik, "Detection, segmentation and recognition of Face and its features using neural network", IEEE transaction paper on image processing.

9. Dr.Mridul Kumar Mathur, Priyanka Bhati, "Face Objects Detection in still images using Viola-Jones Algorithm through MATLAB TOOLS", International Journal of Innovative Research in Computer Communication Engineering.

10. Mohammad Ashraful Islam, Md. AninNaeem, Md.Nazmul Hasan, "Comparision between VIOLA-JONES and KLT Algorithms and error correction of VIOLA-JONES Algorithm", International Journal of Computer Engineering and Applications, Volume XI, Issue V, 2017, www.ijcea.com ISSN 2321-3469. 